\title{
Philosophiques
}

\section{Précis de Sans distinction de race? Une analyse critique du concept de race et de ses effets pratiques}

\section{Magali Bessone}

Volume 40, numéro 2, automne 2013

URI : https://id.erudit.org/iderudit/1023705ar

DOI : https://doi.org/10.7202/1023705ar

Aller au sommaire du numéro

Éditeur(s)

Société de philosophie du Québec

ISSN

0316-2923 (imprimé)

1492-1391 (numérique)

Découvrir la revue

Citer ce document

Bessone, M. (2013). Précis de Sans distinction de race ? Une analyse critique du concept de race et de ses effets pratiques. Philosophiques, 40(2), 457-460.

https://doi.org/10.7202/1023705ar d'utilisation que vous pouvez consulter en ligne.

https://apropos.erudit.org/fr/usagers/politique-dutilisation/ 


\title{
Précis de Sans distinction de race? Une analyse critique du concept de race et de ses effets pratiques
}

\author{
MAGALI BESSONE \\ UFR de philosophie, Université de Rennes 1 \\ magali.bessone-luquet@univ-rennesı.fr
}

L'objet de ce livre est double: il s'agit d'une part de défendre une position épistémique et ontologique sur la race; d'autre part, d'en tirer une position normative sur ce que nous devrions faire avec la race. Il importe de préciser d'emblée que mon horizon philosophique normatif est celui de l'égalitarisme: c'est un idéal d'égalité qui motive la réflexion menée ici. Ma conviction est que dans une société idéale il n'y aurait pas d'inégalité ni de domination pour des motifs raciaux, et donc, que les races n'auraient pas lieu d'être, ni d'être pensées. Cependant, je suis également convaincue que la philosophie politique ne peut s'offrir le luxe de tirer ses principes normatifs d'une situation idéale élaborée avec des agents abstraits, ou d'une situation de pensée fictive, mais qu'elle doit partir de la formulation et de l'élaboration d'un problème dans notre monde non idéal, et produire des normes qui proviennent du problème et tâchent de le résoudre. Les normes en ce sens ne sont pas des principes, mais des instruments permettant de construire une théorie de la justice ou de la démocratie qui s'élaborerait non pas directement et positivement, mais secondairement à partir d'une théorie des difficultés ou des pathologies de la justice et de la démocratie ${ }^{1}$.

Dans cette perspective, la première tâche de la philosophie politique porte sur le diagnostic précis du problème, sur l'identification, à partir de l'observation et de l'interprétation des symptômes, de la pathologie à laquelle il s'agit de trouver un remède. Dans ce travail préalable d'identification, la philosophie normative est nécessairement l'alliée des sciences sociales, en un sens large qui comprend non seulement la sociologie, la psychologie et l'anthropologie, mais également le droit et l'histoire, et dans la mesure où toutes ces sciences fournissent du donné ainsi que des discours interprétatifs, parfois contradictoires, de ce donné.

La seconde tâche de la philosophie politique est d'élaborer la norme — le remède - et d'en tester l'efficacité dans la pratique, visant l'amélioration de l'état général, ce qui implique deux choses: d'une part que la norme soit contextuelle, et toujours susceptible d'être modifiée; d'autre part que nous ayons une vision préalable de ce qu'est un «mieux", sinon une vision parfaite, complète et déterminée de ce qu'est le juste ou le bien. C'est la

1. Cette démarche s'inspire de celle d'Amartya Sen, L'idée de justice, trad. P. Chemla, Paris, Flammarion, 2010 [2009] et d'Elizabeth Anderson, The Imperative of Integration, Princeton, Princeton University Press, 2010.

PHILOSOPHIQUES 40/2 - Automne 2013, p. 457-460 
raison pour laquelle, si la philosophie politique doit bien avoir une visée pratique, il est fondamental de reconnaître qu'il nous est impossible d'éviter le recours à certains idéaux (l'égalité, la non-domination...) et qu'il est donc aussi de notre ressort de nous intéresser à la manière dont nous rencontrons, choisissons et spécifions ces idéaux. Nous pourrons ainsi éviter de formuler des idéaux qui renforcent ou perpétuent les oppressions existantes, et favoriser ceux qui suggèrent des modèles alternatifs.

Le problème dont ce livre tâche de proposer un diagnostic est celui de la persistance, y compris dans des sociétés qui, comme la France, s'interdisent de reconnaître les races, des lignes de fractures sociales, liées tant à des comportements et attitudes individuels qu'à des structures collectives de redistribution et de représentation, pour motifs raciaux. Formuler ce problème exige que l'on s'autorise à parler de races, ou de groupes racialisés, soit à faire usage d'un vocabulaire qui, quoique parfaitement admis dans d'autres contextes nationaux (publics et académiques), est viscéralement tabou en France ${ }^{2}$. Je prétends que l'égalitarisme n'a rien à perdre et tout à gagner à s'emparer de la question: mon livre constitue une tentative de susciter en français une discussion informée et actualisée sur la question raciale. Paradoxalement, s'il remplit sa fonction, ce qu'il soutient devrait apparaittre partiellement absurde ou dépassé à plus ou moins long terme, lorsque la réalité qu'il analyse et la norme qu'il préconise auront été modifiées par les circonstances.

Je défends dans ce livre l'idée d'une construction sociale de la race. Il y a des races d'abord parce qu'il y a une histoire raciale de nos sociétés contemporaines occidentales. On peut tracer, au moins dès le XVIII ${ }^{\mathrm{e}}$ siècle, les modalités d'émergence d'un discours sur la division de l'espèce humaine en groupes repérés par des caractéristiques géographiques et visuelles codant pour des différences morales et cognitives, afin d'asseoir et de justifier le maintien d'inégalités politiques et économiques malgré l'affirmation universaliste d'hommes qui "naissent et demeurent libres et égaux en droit». Une telle enquête généalogique est essentiellement l'objet de mon premier chapitre. Or, comme ce qui a été construit n'a jamais été déconstruit, je défends par suite l'idée que les races, quoique construites historiquement et donc contingentes, existent toujours, et qu'il faut tenir compte de leurs modalités d'existence et de leurs effets actuels afin de lutter contre le(s) racisme(s), autrement dit afin de parvenir in fine à leur déconstruction. Les races existent

2. La récente loi votée à l'Assemblée nationale visant la suppression du mot "race » dans la législation française témoigne de la prégnance de cette position républicaine. Voici en quels termes Alfred Marie-Jeanne, le rapporteur de la loi, introduisait la discussionle I6 mai 20I3: « «Le concept de race, chacun le reconnaît, a servi de fondement aux pires idéologies et a conduit à la mort de millions d'êtres humains. Ce concept scientifiquement aberrant n'a pas sa place dans l'ordre juridique, même si c'est pour condamner toute discrimination fondée sur une prétendue race. [...] Vouloir maintenir à tout prix le mot "race ", n'est-ce pas en effet admettre implicitement son existence?" 
comme systèmes de pouvoir et de domination indexés sur des marqueurs visuels que l'on a appris à considérer comme signifiants.

Ainsi, il y a des races parce qu'il y a des discours, des actes, des comportements, mais aussi des structures sociales et institutionnelles qui racialisent, et qui racialisent certains individus plus que d'autres (cette racialisation n'est absolument pas un phénomène uniforme, symétrique ni égalitaire). De plus, les «identités » ainsi produites sont toujours plus ou moins racialisées, selon le type d'interaction en jeu, sans qu'il y ait quoi que ce soit de fixe, de naturel ou de définitif dans ce qui est ainsi produit - autrement dit, le concept de race ici ne doit surtout pas être entendu dans une acception essentialiste. C'est tout l'enjeu de la discussion que je mène aux chapitres deux, trois et quatre sur le sens qu'il faut accorder au constructivisme, en opposant son projet épistémique d'une part à la tentation de faire de la race une question "naturelle», objet de la biologie, et d'autre part à la tentative de réduire la race à une question "culturelle ", objet de l'anthropologie ou du multiculturalisme. Par contraste, je soutiens que la race n'a d'existence et de sens que dans le domaine socio-politique - il est illusoire de croire que la science (génétique) pourra à elle seule résoudre le problème du racisme, car ce n'est pas le lieu où le problème se constitue, tout comme il est illusoire de croire que le glissement ou la traduction de la race en culture pourra à elle seule fournir une positivité au concept et créer un monde post-raciste où règnerait l'égalité raciale dans la reconnaissance partagée de la «diversité culturelle».

La question ontologique et la question normative sont ainsi indissociables. Admettre que la race est socialement construite, qu'elle ne signifie pas la même chose dans des contextes sociaux et politiques différents - donc que «l'expérience vécue du Noir» de Frantz Fanon et le "problème noir » de W. E. B. Du Bois ${ }^{3}$, en dépit des apparences, ne parlent pas de la même race -, permet de comprendre pourquoi aux chapitres cinq et six, je défends les linéaments d'une position normative "transformative", morale et politique. Je propose de rendre explicites les catégorisations raciales souvent à l'œuvre de manière masquée ou invisible dans les esprits et les institutions, en pariant sur les effets égalisateurs et émancipateurs de leur usage. Après avoir montré comment les racismes existent au pluriel et dans une dialectique de renforcement entre les attitudes individuelles et les institutions politiques, je montre comment ni le multiculturalisme, ni le républicanisme - tous deux «aveuglés » à la question raciale quoique pour des motifs radicalement distincts ni l'égalitarisme libéral dans sa version idéale, ne peuvent apporter de réponse satisfaisante au problème des injustices raciales. Seule une position politique ancrée dans la prise de conscience et la formulation préalables du problème peut s'y confronter de manière féconde. Sur ce point, le livre se contente

3. Frantz Fanon, Peau noire, masques blancs, Paris, Seuil, I952, et W. E. B. Du Bois, Les âmes du peuple noir, I903; trad. M. Bessone, Paris, La Découverte, 2007. 
d'indiquer des pistes, qui seront à poursuivre ailleurs. Un mot seulement: bien entendu, il ne s'agit pas de figer des utilisations délétères et oppressives des races en grille intangible d'interprétation des relations sociales et de redistribution des biens sociaux. La catégorisation raciale doit être utilisée de manière négociable, ad hoc, selon les problèmes qu'elle permettrait d'exposer et de traiter, et comme ressource politique permettant aux minorités racialisées de porter un discours de revendication dans les lieux publics pertinents. C'est bien un usage stratégique à visée déconstructive que je recommande; mon livre a l'ambition d'en fournir le soubassement théorique. 